\title{
REPRESENTING ERGODIC FLOWS AS FLOWS BUILT UNDER FUNCTIONS WITH FINITE RANGE
}

\author{
ROBIN FELLGETT
}

\begin{abstract}
It is shown, using a result of Rudolph, that any cross-section of an ergodic flow whose return-time function is bounded and bounded away from zero is isomorphic to a cross-section whose return-time function has finite range. A weaker result holds if the boundedness conditions are removed.
\end{abstract}

Introduction-Flows with eigenvalues. In this paper we consider an ergodic flow, $\left\{T_{t}: \Omega \rightarrow \Omega: t \in \mathbf{R}\right\}$, on a Lebesgue probability space. A recurring problem in ergodic theory is to describe such flows in the simplest possible way. Such a way involves the construction known as a flow built under a function or special flow, $(T, f)$. Here $T: X \rightarrow X$ is an automorphism of a Lebesgue space, $f$ a positive real function on $X$ and $\Omega=\{(x, r) \in X \times \mathbf{R}$ : $0 \leqslant r<f(x)\}$. Full details are described in Rohlin's survey article [1]. Ambrose [2], see also his paper with Kakutani [3], showed that any ergodic flow is isomorphic to a special flow $(T, f)$ where $f$ is bounded and bounded away from zero. Quite recently Rudolph proved the following, much stronger, theorem.

THEOREM (RUDOLPH [4]). Given any two positive real numbers, $p$ and $q$, such that $p q^{-1}$ is irrational and an ergodic flow, $\left\{T_{t}\right\}$, there exists an automorphism $S: Y \rightarrow Y$ and a partition of $Y$ into two sets, $A$ and $B$, of positive measure such that $\left\{T_{t}\right\}$ is isomorphic to $\left(S, p \chi_{A}+q \chi_{B}\right)$.

(In fact Rudolph's result says even more, which does not concern us here.)

If we have a special flow $(T, f)$ of the type considered by Ambrose then Rudolph's result does not tell us anything about the relationship between $S$ and $T$ except, of course, that they are equivalent in the sense of Kakutani [5]. Below we show, as a consequence of Rudolph's work, that in this case $(T, f)$ is isomorphic to $(T, g)$ where $g$ has finite range. In other words without altering the base transformation it is possible to make the function quite simple. If $f$ is unbounded then $g$ can be chosen with countable range.

I am grateful to William Parry for discussions about this subject.

Received by the editors June 20, 1977.

AMS (MOS) subject classifications (1970). Primary 28A65.

Key words and phrases. Ergodic flow, flow built under a function, cohomologous functions, time change. 
The symbols $\mathbf{N}, \mathbf{Q}, \mathbf{R}$ and $\mathbf{C}$ denote the natural, rational, real and complex numbers respectively. $\mathbf{N}^{+}$and $\mathbf{R}^{+}$denote the positive elements of $\mathbf{N}$ and $\mathbf{R}$. $\chi_{A}$ denotes the characteristic function of a set $A$ and $R(z)$ the real part of $z \in \mathbf{C}$. $\exp (r)$ will be $e^{2 \pi i r}$ and $\log$ the measurable function; $\log (\exp (r))=$ the fractional part of $r$. An eigenvalue, $\alpha \in \mathbf{R}$, and eigenfunction $\theta \in L^{2}(\Omega)$ for the flow satisfy $\theta\left(T_{t} \omega\right)=\exp (\alpha t) \theta(\omega)$. Without loss of generality we may assume this relation holds for every $\omega$ and every $t$ [1]. Two measurable functions, $f$ and $g$, are cohomologous with respect to an automorphism $T$, written $f \sim g$, if there exists a measurable function $h$ so that $f-g=h T-h$ a.e. This is useful as by considering the transformation $(x, r) \rightarrow T_{R(h(x))}(x, r)$ one can show:

THEOREM (GUREVIČ [6]). If $f \sim g$ then $(T, f)$ is isomorphic to $(T, g)$.

We start with a lemma about flows which are not weak mixing.

Lemma 1. Let $(T, f)$ be an ergodic flow with eigenvalue $\alpha$. Then, if $f$ is bounded away from zero, there exists an integer $N>0$ and a function, $g$ : $X \rightarrow \mathbf{R}^{+}$, such that $g(x) \in \mathbf{N}^{+}(N \alpha)^{-1}$ and $(T, f)$ is isomorphic to $(T, g)$. If $f$ is bounded then so is $g$.

Proof. We have $\theta\left(T_{t} \omega\right)=\exp (\alpha t) \theta(\omega)$. Let $H: X \rightarrow \mathbf{C}$ be defined by $H(x)=\theta(x, 0)$ so $H(T x)=\exp (\alpha f(x)) H(x)$. $|\theta(\omega)|=1$ except on an invariant set of measure zero so $|H(x)|=1$ a.e. and

$$
M(x)+\log H(T x)=\alpha f(x)+\log H(x) \text { a.e., }
$$

where $M$ is a measurable integer valued function, which may be set equal to one where not already defined. I.e. $f+(\log H) \alpha^{-1}-(\log H T) \alpha^{-1}=M \alpha^{-1}$ a.e.

Since $(\log H) \alpha^{-1}$ is bounded and $f$ is bounded away from zero there exists an integer $N>0$ such that:

$$
\sum_{i=0}^{N-1} f T^{i}+(\log H) \alpha^{-1}-\left(\log H T^{N}\right) \alpha^{-1}=\sum_{i=0}^{N-1}\left(M T^{i}\right) \alpha^{-1}>0 .
$$

As $\sum_{i=0}^{N-1} f T^{i} \sim N f$ we have $f \sim g$, where

$$
g(x)=\sum_{i=0}^{N-1}\left(M T^{i}(x)\right)(N \alpha)^{-1}
$$

has the required properties.

$f=g+h T-h$ a.e. where if $f$ is bounded $h$ is also bounded. The final statement follows so the lemma is proved, using Gurevič's theorem.

2. The main result. We now turn to our main result. The reader who is prepared to draw a few pictures will probably be convinced that it ought to be true. Lemma 1 provides a technique for overcoming the technicalities and so provides a straightforward proof of the theorem. 
THEOREM. Let $(T, f)$ be an ergodic flow and $\alpha$ and $\beta$ any two positive real numbers such that $\alpha \beta^{-1}$ is irrational.

(i) If $f$ is bounded away from zero there exists an integer $N>0$ and a function $g: X \rightarrow \mathbf{R}^{+}$, such that $g(x) \in \mathbf{N}(N \alpha)^{-1}+\mathbf{N}(N \beta)^{-1}$ a.e. and $(T, f)$ is isomorphic to $(T, g)$.

(ii) If, in addition, $f$ is bounded then $g$ is also bounded and so has finite range.

(iii) If no boundedness conditions are placed on $f$ then there exists a function $g: X \rightarrow \mathbf{R}^{+}$, such that $g(x) \in \mathbf{Q} \alpha^{-1}+\mathbf{Q} \beta^{-1}$ a.e. and $(T, f)$ is isomorphic to $(T, g)$.

Proof of (i). By Rudolph's theorem $(T, f)$ is isomorphic to $\left(S, \alpha^{-1} \chi_{A}+\right.$ $\left.\beta^{-1} \chi_{B}\right)$. Denote the second special flow by $T_{i}: \Omega \rightarrow \Omega$. We use some standard notation for time changes (see [7] for example). Time change $\left\{T_{t}\right\}$ to $S_{t}(\omega)=$ $T_{h(t, \omega)}(\omega)$, where

$$
\lim t^{-1} h(t,(y, r))= \begin{cases}1, & y \in A, \\ \beta^{-1} \alpha, & y \in B,\end{cases}
$$

and the limit is taken as $t$ decreases to zero. The flow $\left\{S_{t}\right\}$ is the special flow $\left(S, \alpha^{-1}\right)$ and is isomorphic to a special flow $\left(T, f_{1}\right)$, where $f_{1}$ is also bounded away from zero. Since $\left(S, \alpha^{-1}\right)$ has an eigenvalue $\alpha$ it is isomorphic, by Lemma 1 , to a special flow $\left(T, f_{2}\right)$. Here there exists $N>0$ such that $f_{2}(x) \in \mathbf{N}^{+}(N \alpha)^{-1}$ a.e.

Set $G=N \alpha f_{2}$ and consider the tower transformation (see [8] for example) $\tilde{T}=T^{G}$, which acts on the space $\tilde{X}=\{(x, n): x \in X, 0 \leqslant n<G(x)\}$, and the tower transformation $\tilde{S}=S^{N}$, which acts on $\tilde{Y}=\{(y, n): y \in Y, 0 \leqslant n$ $<N\}$. If $\iota:\left[0, \alpha^{-1}\right) \rightarrow\left[0, \alpha^{-1}\right)$ is the identity transformation then $\tilde{S} \times \iota$ is the flow element $S_{(N \alpha)^{-1}}$ which is isomorphic, via $U: \tilde{Y} \times\left[0, \alpha^{-1}\right) \rightarrow \tilde{X} \times$ $\left[0, \alpha^{-1}\right)$ say, to $\tilde{T} \times \iota . \tilde{S}$ is ergodic so $\iota$ is the factor of $\tilde{S} \times \iota$ corresponding to the $\sigma$-algebra of all $\tilde{S} \times \iota$ invariant sets. $\iota$ is a similar factor of $\tilde{T} \times \iota$ so for a.e. $r \in\left[0, \alpha^{-1}\right) ; U(\tilde{y}, r)=\left(\tau_{r}(\tilde{y}), r^{\prime}\right)$. Thus we conclude there is an isomorphism, $\tau: \tilde{Y} \rightarrow \tilde{X}$ of $\tilde{S}$ and $\tilde{T}$ and $\left(S, \alpha^{-1}\right)=\left(\tilde{S},(N \alpha)^{-1}\right)$ is isomorphic to $\left(T,(N \alpha)^{-1}\right)=\left(T, f_{2}\right)$ under the isomorphism $(\tilde{y}, r) \rightarrow(\tau \tilde{y}, r)$. Applying the inverse time change $T_{t}(\omega)=S_{k(t, \omega)}(\omega)$, where $t=k(h(t, \omega), \omega)$, we see $\left\{T_{t}\right\}$ is isomorphic to $\left(\tilde{T}, g_{1}\right)$, where $g_{1}(\tilde{x}) \in\left\{(N \alpha)^{-1},(N \beta)^{-1}\right\}$ a.e. Now $\left(T, g_{1}\right)=$ $(T, g)$, where $g$ is of the form stated in the theorem and (i) is proved.

Proof of (ii). Exactly as (i), using the final statement in Lemma 1.

Proof of (iii). Choose any $\varepsilon>0$ so that the set $A=\{x: f(x)>\varepsilon\}$ has positive measure. $T$ is a tower over the induced transformation $T_{A}$, say $T=\left(T_{A}\right)^{F}$, and $(T, f)=\left(T_{A}, f_{1}\right)$ where $f_{1}>\varepsilon$. We have $\left(T_{A}, f_{1}\right)$ isomorphic to $\left(T_{A}, g_{1}\right)$, where $g_{1}$ is of the form described in (i). Let, for all $x \in X$, $m(x)=\min \left\{m \in N: T^{-m}(x) \in A\right\}$. Now define

$$
g(x)=g_{1}(x)\left(F\left(T^{-m(x)}(x)\right)\right)^{-1} .
$$

Then $\left(T_{A}, g_{1}\right)=(T, g)$ and the theorem is proved. 
REMARK. The same technique, using Lemma 1, provides a simple proof of Kakutani's theorem [5].

\section{REFERENCES}

1. V. A. Rohlin, Selected topics in the metric theory of dynamical systems, Uspehi Mat. Nauk 4 (1949), 57-128 = Amer. Math. Soc. Transl. (2) 49 (1966), 171-240.

2. W. Ambrose, Representation of ergodic flows, Ann. of Math. 42 (1941), 723-739.

3. W. Ambrose and S. Kakutani, Structure and continuity of measurable flows, Duke Math. J. 9 (1942), 25-42.

4. D. Rudolph, A 2-valued step coding for ergodic flows, Math. Z. 150 (1976), 201-220.

5. S. Kakutani, Induced measure preserving transformations, Proc. Imp. Acad. Tokyo 19 (1943), 635-641.

6. B. M. Gurevic, Construction of increasing partitions for special flows, Teor. Verojatnost. i Primenen. 10 (1965), 693-712 = Theor. Probability Appl. 10 (1965), 627-645.

7. W. Parry, Cocycles and velocity changes, J. London Math. Soc. 5 (1972), 511-516.

8. N. Friedman, Introduction to ergodic theory, Van Nostrand, Princeton, N. J., 1970.

Department of Mathematics, University of Maryland, College Park, Maryland 20742 